\title{
OPEN Multiple nutritional and gut microbial factors associated with allergic rhinitis: the Hitachi Health Study
}

\author{
Yukari Sahoyama ${ }^{1 \bowtie}$, Fumiaki Hamazato ${ }^{1}$, Manabu Shiozawa ${ }^{1}$, Tohru Nakagawa ${ }^{2}$, \\ Wataru Suda ${ }^{3}$, Yusuke Ogata ${ }^{3}$, Tsuyoshi Hachiya ${ }^{4}$, Eiryo Kawakami ${ }^{5,6}$ \& Masahira Hattori ${ }^{3,7}$
}

Several studies suggest the involvement of dietary habits and gut microbiome in allergic diseases. However, little is known about the nutritional and gut microbial factors associated with the risk of allergic rhinitis (AR). We recruited 186 participants with symptoms of AR and 106 control subjects without symptoms of AR at the Hitachi Health Care Center, Japan. The habitual consumption of 42 selected nutrients were examined using the brief-type self-administered diet history questionnaire. Faecal samples were collected and subjected to amplicon sequencing of the $16 \mathrm{~S}$ ribosomal RNA gene hypervariable regions. Association analysis revealed that four nutrients (retinol, vitamin A, cryptoxanthin, and copper) were negatively associated with AR. Among 40 genera examined, relative abundance of Prevotella and Escherichia were associated with AR. Furthermore, significant statistical interactions were observed between retinol and Prevotella. The age- and sex-adjusted odds of AR were 25 -fold lower in subjects with high retinol intake and high Prevotella abundance compared to subjects with low retinol intake and low Prevotella abundance. Our data provide insights into complex interplay between dietary nutrients, gut microbiome, and the development of AR.

Allergic rhinitis (AR) is a symptomatic disorder of the nose caused by immunoglobulin E (IgE)-mediated reactions against inhaled allergens ${ }^{1,2}$. The classical symptoms of AR are nasal itching, sneezing, rhinorrhoea, and nasal congestion, and ocular symptoms are also frequent ${ }^{3}$. Over 500 million people worldwide are suffering from $\mathrm{AR}^{1}$. The burden of disease related to $\mathrm{AR}$ is substantial due to reduced patients' quality of life (QoL), lower performance at work and school, poorer sleep quality and cognitive function, and increased irritability and fatigue $^{3,4}$. Moreover, AR is a risk factor for asthma ${ }^{3}$. Despite recognition of AR as a global health problem, its aetiological risk factors have yet to be fully understood ${ }^{1}$.

The prevalence of allergic diseases, including AR and asthma, has increased in many countries, and changes in dietary habits may be one of the factors responsible for this trend ${ }^{5}$. Diet is an important source of nutrients that may modulate the risk of allergic diseases ${ }^{6}$. For instance, systemic oxidative stress enhances inflammatory responses relevant to allergy; and therefore, antioxidants may play a key role in the prevention of allergic diseases $^{7}$. Furthermore, epidemiological studies have suggested that fruits and vegetables that contain several antioxidants protect against allergic diseases ${ }^{8}$. Therefore, researchers have intensively investigated the nutrients exhibiting a protective (or adverse) effect on the development of allergic diseases, such as asthma ${ }^{5-8}$. However, little is known about the relationship between nutrients and the risk of AR.

Recent studies have suggested that gut microbiota may be involved in the pathogenesis of allergic diseases. The gut microbiota is mainly composed of bacteria, and its composition is highly dynamic and influenced by dietary habits of their host ${ }^{9}$. The gut microbiota produces bioactive metabolites from dietary materials ${ }^{10}$. These metabolites influence host immune responses through the interplay with regulatory $\mathrm{T}$ (Treg) and dendritic cells. Therefore, the composition of the intestinal microbiota potentially links with the development of allergic diseases $^{9-11}$.

\footnotetext{
${ }^{1}$ Technology Innovation Div., Hitachi High-Tech Corporation, Business Tower, Toranomon Hills, 1-17-1 Minato-ku, Toranomon, Tokyo 105-6409, Japan. ${ }^{2}$ Hitachi Health Care Center, Hitachi Ltd., Ibaraki, Japan. ${ }^{3}$ Laboratory for Microbiome Sciences, RIKEN Center for Integrative Medical Sciences, Yokohama, Japan. ${ }^{4}$ Genome Analytics Japan Inc., Tokyo, Japan. ${ }^{5}$ Medical Sciences Innovation Hub Program, RIKEN, Yokohama, Japan. ${ }^{6}$ Artificial Intelligence Medicine, Graduate School of Medicine, Chiba University, Chiba, Japan. ${ }^{7}$ Graduate School of Advanced Science and Engineering, Waseda University, Tokyo, Japan. ${ }^{\circledR}$ email: yukari.sahoyama.vp@hitachi-hightech.com
} 
In the present cross-sectional study, we investigated the relationship between daily nutritional intake, gut microbiome composition, and self-reported AR symptoms in middle-aged workers. Our aim was three-fold: (i) to suggest which nutrients are associated with AR; (ii) to search for microbes whose abundances are associated with AR; and (iii) to examine whether nutrients and microbes have a combinatorial effect on AR. Various dietary components are metabolised by gut microbiome enzymes; and therefore, the effect of dietary nutrients on the odds of AR may be modulated by intestinal microbiota.

\section{Methods}

Study subjects. The study participants were recruited at the Hitachi Health Care (HHC) Center, where annual health examinations of employees and their spouses from 35 affiliated companies $(\sim 38,000$ workers in total) are performed. Most visitors at the HHC Center were employed by electronics-related companies and performed generally representative (not industry-specific) jobs, including general affairs, design, research and development, sales, and manufacturing. The characteristics of the visitors were described previously ${ }^{12-14}$. They have continuously undergone annual comprehensive health examinations, including cardiovascular and cancer screening. Therefore, we could select visitors based on the results of their previous health examinations. Previous visitors without a history of serious illness (cancer, cerebrovascular disease, or myocardial infarction) and unhealthy risk factors (such as high blood pressure, high glucose level, or abnormal lipid levels) were invited to participate in this study by e-mail. The number of enrolled volunteers was 301 , and they were asked at visit reservation whether they had symptoms of seasonal or perennial AR. They re-visited the HHC Center for their annual health check-up from September 2018 to February 2019. The study participants were asked to complete a questionnaire on their dietary habits and symptoms of AR. In addition, stool samples were collected. Nine subjects were excluded due to withdrawal of consent $(n=2)$ or their inconsistent report of AR symptoms in the questionnaire compared to that at visit reservation $(n=7)$. Finally, 186 participants with symptoms of AR and 106 control subjects without symptoms of AR were included in the study. This study was reviewed and approved in advance by the Hitachi Hospital Group Ethics Committee (Approved No. 2018-5, 2019-10, and 2020-88), the Institutional Review Board of the Hitachi Ltd. (Approved No. 220-1 and 238-1), and the Research Ethics Committee of RIKEN (Approved No. H30-5). All participants provided written informed consent. This study was conducted according to the principles of the Declaration of Helsinki.

Nutritional intake. Nutritional consumption data for the study participants were obtained using the brieftype self-administered diet history questionnaire (BDHQ). The questionnaire asked about the intake frequency of 58 selected food and beverage items, which are commonly consumed in Japan, in the preceding month ${ }^{15,16}$. The BDHQ does not consider the portion size. Instead, standard portion sizes were defined according to recipe books for Japanese diets ${ }^{15,16}$. Based on the standard tables of food composition in Japan revised in $2010^{17}$, crude estimates for dietary intake of total energy and 42 selected nutrients were calculated. Energy adjustment was performed using the density method ${ }^{16,18}$. Adjusted values for energy-providing nutrients were calculated by dividing the amount of energy obtained from each nutrient by the total energy intake. Adjusted values for nonenergy-providing nutrients were defined as the amount of weight of each nutrient per $10 \mathrm{MJ}$ of dietary energy intake.

Gut microbiome. Fresh faecal samples were collected in plastic containers containing glass beads (Tomy Seiko) and RNAlater Reagent (Life Technologies Japan). The samples were transported at $4{ }^{\circ} \mathrm{C}$ to the laboratory. In the laboratory, the faecal samples $(\sim 0.2 \mathrm{~g})$ were suspended in $15 \mathrm{~mL}$ phosphate-buffered saline (PBS) buffer and filtered with a 100- $\mu \mathrm{m}$-mesh nylon filter (Corning) to remove human and eukaryotic cells and debris from the faecal sample. The debris on the filter was washed twice with PBS. The bacteria-enriched pellet was obtained by centrifugation of the filtrate at $9000 \times \mathrm{g}$ for $10 \mathrm{~min}$ at $4{ }^{\circ} \mathrm{C}$. The pellets were washed with $35 \mathrm{~mL} \mathrm{PBS}$ once, further washed with TE20 buffer (10 mM Tris-HCl, $20 \mathrm{mM}$ EDTA), and subjected to DNA extraction. Bacterial DNA was isolated and purified from the faecal samples according to enzymatic lysis methods described previously ${ }^{19}$.

The 16S V1-V2 region was amplified using polymerase chain reaction (PCR) with barcoded 27Fmod $\left(5^{\prime}\right.$-agrgtttgatymtggctcag- $\left.3^{\prime}\right)$ and the reverse primer 338R (5'-tgctgcctcccgtaggagt- $\left.3^{\prime}\right)^{19}$. PCR was performed using $50 \mu \mathrm{L}$ of $1 \times$ Ex Taq PCR buffer composed of $10 \mathrm{mM}$ Tris. $\mathrm{HCl}(\mathrm{pH} 8.3), 50 \mathrm{mM} \mathrm{KCl}$, and $1.5 \mathrm{mM} \mathrm{MgCl}_{2}$ in the presence of $250 \mu \mathrm{M}$ dNTPs, 1 unit Ex Taq polymerase (Takara Bio), forward and reverse primers $(0.2 \mu \mathrm{M})$, and $20 \mathrm{ng}$ of template DNA. PCR was performed in a 9700 PCR System (Life Technologies Japan), and the following cycling conditions were used: initial denaturation at $96^{\circ} \mathrm{C}$ for $2 \mathrm{~min}$, followed by 20 cycles of denaturation at $96^{\circ} \mathrm{C}$ for $30 \mathrm{~s}$, annealing at $55^{\circ} \mathrm{C}$ for $45 \mathrm{~s}$, and extension at $72{ }^{\circ} \mathrm{C}$ for $1 \mathrm{~min}$, with a final extension at $72^{\circ} \mathrm{C}$. PCR amplicons were purified using AMPure XP magnetic purification beads (Beckman Coulter) and quantified using the Quant-iT PicoGreen dsDNA Assay Kit (Life Technologies Japan). An equal amount of each PCR amplicon was mixed and subjected to multiplexed amplicon sequencing with MiSeq $(2 \times 300$ paired-end run), according to the manufacturer's instructions.

Two paired-end reads were merged using the fastq-join program based on overlapping sequences. Reads with an average quality value of $<25$ and inexact matches to both universal primers were filtered out. The reads lacking both forward and reverse primer sequences were removed. Filter-passed reads were used after trimming off both primer sequences. Reads having BLAST match lengths $<90 \%$ with the representative sequence in the $16 \mathrm{~S}$ databases (described below) were considered as chimeras and removed. Finally, filter-passed reads were used for further analysis. The $16 \mathrm{~S}$ database was constructed from three publicly available databases: Ribosomal Database Project (RDP) (Release 11, Update 5), CORE (October 13, 2017 updated; http://microbiome.osu.edu/), and a reference genome sequence database obtained from the NCBI FTP site (ftp://ftp.ncbi.nih.gov/genbank/, April 


\begin{tabular}{|l|l|l|l|}
\hline & Allergic rhinitis & Control & $P$-value \\
\hline Number of subjects & 186 & 106 & - \\
\hline Age, years & $49.2 \pm 7.1$ & $50.4 \pm 8.2$ & 0.117 \\
\hline Male, \% & 87.6 & 91.5 & 0.338 \\
\hline Body mass index, kg/m ${ }^{2}$ & $23.6 \pm 3.0$ & $23.7 \pm 3.1$ & 0.706 \\
\hline Systolic blood pressure, $\mathrm{mmHg}$ & $117.9 \pm 11.4$ & $122.4 \pm 12.9$ & 0.006 \\
\hline Diastolic blood pressure, $\mathrm{mmHg}$ & $75.5 \pm 8.5$ & $79.1 \pm 8.9$ & 0.003 \\
\hline HbAlc, \% & $5.6 \pm 0.3$ & $5.6 \pm 0.4$ & 0.241 \\
\hline Serum total cholesterol, $\mathrm{mmol} / \mathrm{L}$ & $5.43 \pm 0.76$ & $5.52 \pm 0.74$ & 0.593 \\
\hline LDL cholesterol, mmol/L & $3.19 \pm 0.68$ & $3.28 \pm 0.67$ & 0.261 \\
\hline HDL cholesterol, $\mathrm{mmol} / \mathrm{L}$ & $1.58 \pm 0.37$ & $1.55 \pm 0.41$ & 0.251 \\
\hline Triglyceride, $\mathrm{mmol} / \mathrm{L}$ & $1.34 \pm 0.96$ & $1.49 \pm 0.87$ & 0.035 \\
\hline
\end{tabular}

Table 1. Characteristics of study participants. Values are shown as mean \pm standard deviation or frequency. $P$-values were calculated using the Wilcoxon rank sum test for continuous variables or Fisher's exact test for discrete variables. No adjustments were made for statistical tests shown in this table. HDL indicates highdensity lipoprotein; LDL, low-density lipoprotein.

2013). From the filter-passed reads, 10,000 high-quality reads per sample were randomly chosen. The total reads (the number of samples $\times 10,000$ ) were then sorted by frequency of redundant sequences and grouped into operational taxonomic units (OTUs) using UCLUST with a sequence identity threshold of $97 \%$. The representative sequences of the generated OTUs were subjected to a homology search against the databases mentioned above using the GLSEARCH program for taxonomic assignments. For assignment at the phylum, genus, and species levels, sequence similarity thresholds of $70 \%, 94 \%$, and $97 \%$, respectively, were applied.

Three measures of alpha-diversity (Chao $1, \mathrm{ACE}$, and Shannon's index) ${ }^{20}$ were calculated based on the OTUlevel bacterial composition. The comparison of overall microbiome structure between subject groups was conducted by a permutational multivariate analysis of variance (PERMANOVA) using R software (v4.1.1). The genus-level relative abundance was represented as a percentage. Genera with a mean relative abundance of $\geq 0.2 \%$ were considered for statistical analyses.

Statistical analysis. Differences in clinical characteristics between the AR and control groups were assessed using the Wilcoxon rank sum test for continuous variables or Fisher's exact test for discrete variables. Similarly, potential associations of nutritional and microbial variables with AR were tested using the Wilcoxon rank sum test. For nutrients and microbial genera associated with AR, age- and sex-adjusted logistic regression analysis was performed. Analysis of the receiver operating characteristic (ROC) curve and area under the curve (AUC) was performed with 'pROC' R package ${ }^{21}$. In the logistic regression analysis, nutritional and microbial variables were classified into four categories based on quartiles (Q1-Q4). The same definition of variable categories was also used in multivariate analyses that incorporated age, sex, multiple nutrients, and/or multiple microbial genera. Statistical interaction was tested by adding a multiplicative interaction term in an age- and sex-adjusted logistic regression model and by using the chi-squared test for the comparison with and without the interaction term. Two-sided $P<0.05$ was considered statistically significant, and two-sided $P<0.1$ was regarded as marginally significant in all analyses. All statistical analyses were performed using the R software 3.6.1 (R Foundation for Statistical Computing).

\section{Results}

Clinical characteristics of study participants. The number of subjects who reported the symptoms of AR was 186, and the number of control subjects was 106 (Table 1). The mean age of the subjects with AR and controls was 49.2 and 50.4, respectively. Almost $90 \%$ of the subjects were male in both groups. Subjects with AR symptoms had significantly lower blood pressure and triglyceride levels than controls $(P=0.006$ for systolic blood pressure; $P=0.003$ for diastolic blood pressure; and $P=0.035$ for triglyceride). No significant differences between the groups were observed in body mass index, $\mathrm{HbA1c}$, serum total cholesterol, low-density lipoprotein cholesterol, and high-density lipoprotein cholesterol.

Nutritional intake and AR. The total dietary energy was similar between the AR and control groups (mean of 7819 and $7966 \mathrm{~kJ} /$ day for the AR and control groups, respectively) (Table 2). Among the 42 selected nutrients, energy-adjusted intakes of 4 nutrients (retinol, vitamin A, cryptoxanthin, and copper) were significantly different between AR and control groups ( $P=0.001,0.003,0.007$, and 0.023 , respectively; Wilcoxon rank sum test).

The AR group consumed a lower level of retinol than controls (Fig. 1a). A clear dose-dependent trend was observed in the association between retinol and AR (Fig. 1b). Compared to the first quartile (Q1) of retinol intake as reference, the age- and sex-adjusted odds ratios for Q2, Q3, and Q4 were $0.83,0.69$, and 0.22 , respectively $(P$ for trend $=0.0004)$. Q4 had a significantly lower odds of AR than Q1 $(P=0.0001)$, whereas Q2 and Q3 did not. Similar dose-dependent trends were observed in the association of vitamin A and cryptoxanthin with AR (Fig. 1c-f). 


\begin{tabular}{|c|c|c|c|c|}
\hline & Unit & Allergic rhinitis $(n=186)$ & Control $(n=106)$ & $P$-value \\
\hline Total energy & $\mathrm{kJ} /$ day & $7819 \pm 2100$ & $7966 \pm 2135$ & 0.576 \\
\hline Protein & $\%$ energy & $14.24 \pm 2.29$ & $14.72 \pm 2.36$ & 0.152 \\
\hline Fat & $\%$ energy & $24.87 \pm 5.24$ & $25.28 \pm 5.44$ & 0.794 \\
\hline Saturated fat & $\%$ energy & $6.51 \pm 1.82$ & $6.38 \pm 1.63$ & 0.644 \\
\hline Monounsaturated fat & $\%$ energy & $8.96 \pm 2.11$ & $9.14 \pm 2.23$ & 0.767 \\
\hline Polyunsaturated fat & $\%$ energy & $6.26 \pm 1.22$ & $6.56 \pm 1.37$ & 0.107 \\
\hline n-6 polyunsaturated fat & $\%$ energy & $5.04 \pm 1.00$ & $5.26 \pm 1.11$ & 0.161 \\
\hline n-3 polyunsaturated fat & $\%$ energy & $1.20 \pm 0.30$ & $1.28 \pm 0.36$ & 0.125 \\
\hline $\mathrm{n}-3$ polyunsaturated fat of marine origin ${ }^{\mathrm{a}}$ & $\%$ energy & $0.39 \pm 0.18$ & $0.41 \pm 0.22$ & 0.388 \\
\hline Eicosapentaenoic acid & $\%$ energy & $0.13 \pm 0.07$ & $0.14 \pm 0.08$ & 0.403 \\
\hline Docosahexaenoic acid & $\%$ energy & $0.22 \pm 0.10$ & $0.23 \pm 0.12$ & 0.373 \\
\hline a-Linolenic acid & $\%$ energy & $0.77 \pm 0.19$ & $0.82 \pm 0.21$ & 0.118 \\
\hline Cholesterol & $\mathrm{mg} / 10 \mathrm{MJ}$ & $445 \pm 153$ & $458 \pm 178$ & 0.752 \\
\hline Carbohydrate & $\%$ energy & $51.61 \pm 8.41$ & $51.89 \pm 6.94$ & 0.914 \\
\hline Total dietary fibre & $\mathrm{g} / 10 \mathrm{MJ}$ & $13.61 \pm 3.79$ & $14.44 \pm 3.88$ & 0.060 \\
\hline Soluble dietary fibre & $\mathrm{g} / 10 \mathrm{MJ}$ & $3.44 \pm 1.06$ & $3.61 \pm 1.14$ & 0.370 \\
\hline Insoluble dietary fibre & $\mathrm{g} / 10 \mathrm{MJ}$ & $9.77 \pm 2.68$ & $10.29 \pm 2.57$ & 0.072 \\
\hline Alcohol & $\%$ energy & $7.78 \pm 9.05$ & $6.59 \pm 6.97$ & 0.995 \\
\hline Retinol & $\mu \mathrm{g} / 10 \mathrm{MJ}$ & $484 \pm 347$ & $709 \pm 909$ & 0.001 \\
\hline Vitamin A (retinol equivalent) ${ }^{\mathrm{b}}$ & $\mu \mathrm{g} / 10 \mathrm{MJ}$ & $779 \pm 415$ & $1013 \pm 939$ & 0.003 \\
\hline a-Carotene & $\mu \mathrm{g} / 10 \mathrm{MJ}$ & $389 \pm 323$ & $366 \pm 306$ & 0.351 \\
\hline$\beta$-Carotene & $\mu \mathrm{g} / 10 \mathrm{MJ}$ & $3154 \pm 2145$ & $3240 \pm 2006$ & 0.570 \\
\hline$\beta$-Carotene equivalent ${ }^{\mathrm{c}}$ & $\mu \mathrm{g} / 10 \mathrm{MJ}$ & $3488 \pm 2293$ & $3603 \pm 2191$ & 0.523 \\
\hline Cryptoxanthin & $\mu \mathrm{g} / 10 \mathrm{MJ}$ & $274 \pm 271$ & $355 \pm 291$ & 0.007 \\
\hline a-Tocopherol & $\mathrm{mg} / 10 \mathrm{MJ}$ & $8.36 \pm 2.01$ & $8.77 \pm 2.08$ & 0.130 \\
\hline Vitamin $\mathrm{K}$ & $\mu \mathrm{g} / 10 \mathrm{MJ}$ & $382 \pm 182$ & $417 \pm 190$ & 0.088 \\
\hline Thiamine & $\mathrm{mg} / 10 \mathrm{MJ}$ & $0.90 \pm 0.18$ & $0.94 \pm 0.19$ & 0.260 \\
\hline Riboflavin & $\mathrm{mg} / 10 \mathrm{MJ}$ & $1.60 \pm 0.42$ & $1.65 \pm 0.44$ & 0.413 \\
\hline Niacin & $\mathrm{mg} / 10 \mathrm{MJ}$ & $21.85 \pm 5.17$ & $22.77 \pm 5.25$ & 0.222 \\
\hline Vitamin B6 & $\mathrm{mg} / 10 \mathrm{MJ}$ & $1.52 \pm 0.33$ & $1.56 \pm 0.32$ & 0.271 \\
\hline Vitamin B12 & $\mu \mathrm{g} / 10 \mathrm{MJ}$ & $10.52 \pm 4.67$ & $11.77 \pm 5.97$ & 0.063 \\
\hline Folate & $\mu \mathrm{g} / 10 \mathrm{MJ}$ & $388 \pm 142$ & $413 \pm 139$ & 0.088 \\
\hline Pantothenic acid & $\mathrm{mg} / 10 \mathrm{MJ}$ & $7.98 \pm 1.53$ & $8.34 \pm 1.67$ & 0.110 \\
\hline Vitamin C & $\mathrm{mg} / 10 \mathrm{MJ}$ & $119 \pm 58$ & $126 \pm 52$ & 0.151 \\
\hline Sodium & $\mathrm{mg} / 10 \mathrm{MJ}$ & $5344 \pm 1049$ & $5529 \pm 995$ & 0.101 \\
\hline Potassium & $\mathrm{mg} / 10 \mathrm{MJ}$ & $2963 \pm 727$ & $3070 \pm 729$ & 0.215 \\
\hline Calcium & $\mathrm{mg} / 10 \mathrm{MJ}$ & $613 \pm 232$ & $621 \pm 203$ & 0.367 \\
\hline Magnesium & $\mathrm{mg} / 10 \mathrm{MJ}$ & $310 \pm 57$ & $321 \pm 58$ & 0.149 \\
\hline Phosphorus & $\mathrm{mg} / 10 \mathrm{MJ}$ & $1269 \pm 230$ & $1302 \pm 222$ & 0.181 \\
\hline Iron & $\mathrm{mg} / 10 \mathrm{MJ}$ & $9.29 \pm 2.24$ & $9.77 \pm 2.15$ & 0.063 \\
\hline Zinc & $\mathrm{mg} / 10 \mathrm{MJ}$ & $10.10 \pm 1.41$ & $10.48 \pm 1.42$ & 0.053 \\
\hline Copper & $\mathrm{mg} / 10 \mathrm{MJ}$ & $1.41 \pm 0.24$ & $1.48 \pm 0.22$ & 0.023 \\
\hline Manganese & $\mathrm{mg} / 10 \mathrm{MJ}$ & $4.03 \pm 1.24$ & $3.95 \pm 1.09$ & 0.793 \\
\hline
\end{tabular}

Table 2. Energy-adjusted nutrient intakes and allergic rhinitis. Values are shown as the mean \pm standard deviation. $P$-values were calculated using the Wilcoxon rank sum test. No adjustments were made for statistical tests shown in this table. ${ }^{a}$ Sum of eicosapentaenoic acid, docosapentaenoic acid, and docosahexaenoic acid ${ }^{16}$. ${ }^{\mathrm{b}}$ Sum of retinol, $\beta$-carotene $/ 12$, $\alpha$-carotene $/ 24$, and cryptoxanthin $/ 24^{16}$. ${ }^{\mathrm{C}}$ Sum of $\beta$-carotene, $\alpha$-carotene $/ 2$, and cryptoxanthin $/ 2^{16}$.

There was a reverse J-shaped relationship between copper and AR (Fig. 1g, h). Compared to Q1 of copper intake as reference, the age- and sex-adjusted odds ratios for Q2, Q3, and Q4 were 0.38, 0.32, and 0.58, respectively. Q2 and Q3 showed a significantly lower odds of AR than Q1, whereas Q4 did not.

A multivariate analysis incorporating age, sex, and the four nutrients associated with AR (i.e., retinol, vitamin A, cryptoxanthin, and copper) suggested that a high level of retinol intake (Q4), a high level of cryptoxanthin (Q4), and intermediate levels of copper intake (Q2 and Q3) were independently associated with decreased odds of AR $(P<0.05$; Supplementary Table S1). Vitamin A was not significantly associated with AR in the multivariate 
a
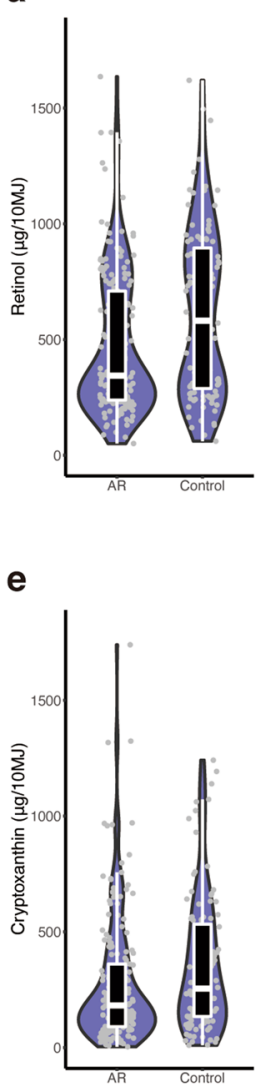

b

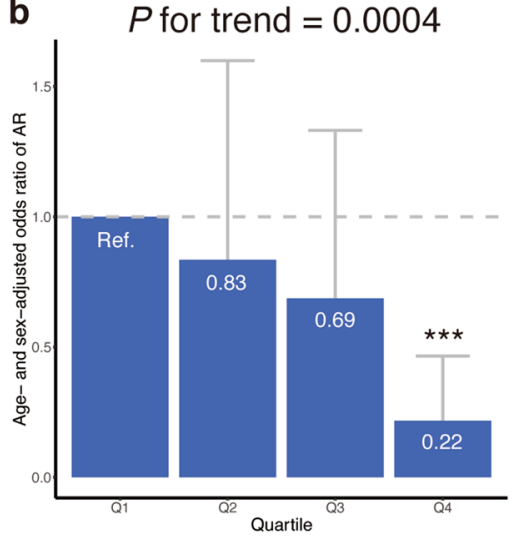

f

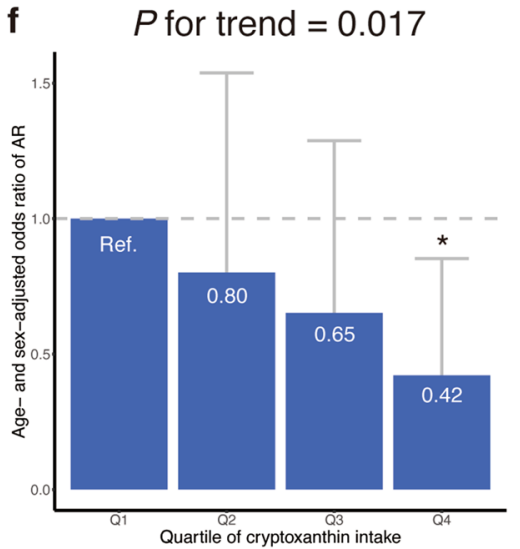

C

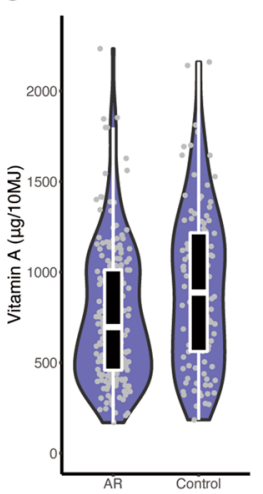

g

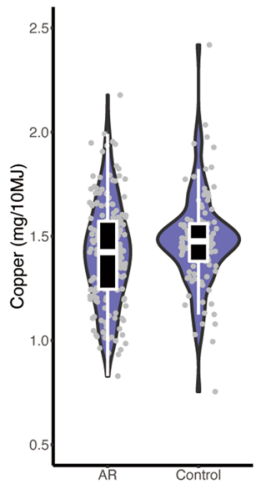

d

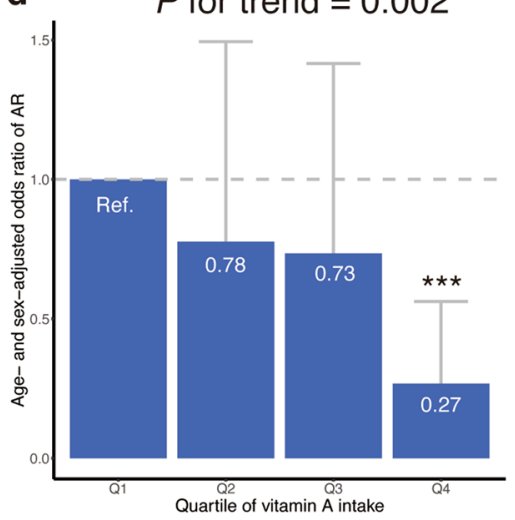

h $\quad P$ for trend $=0.048$

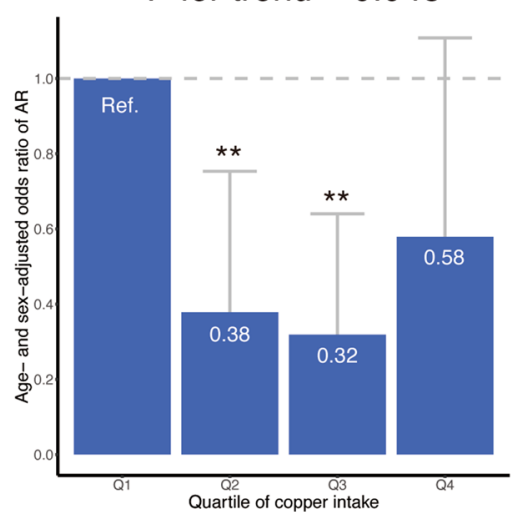

Figure 1. Nutrients associated with allergic rhinitis. The distribution of energy-adjusted nutritional intake is shown for (a) retinol, (c) vitamin A, (e) cryptoxanthin, and (g) copper. Outlier data points are excluded from these plots. The age- and sex-adjusted odds ratio of allergic rhinitis is presented for (b) retinol, (d) vitamin A, (f) cryptoxanthin, and (h) copper. Error bars indicate $95 \%$ confidence interval. ' ${ }^{\prime}$ indicates $P<0.05$; ‘**), $P<0.01$; and '***', $P<0.001$. AR, allergic rhinitis; Q, quartile; Ref., reference group.

analysis $(P>0.05)$, possibly because the intake levels of retinol and vitamin A were highly correlated $(R=0.96$; Supplementary Fig. S1).

The energy-adjusted intake levels of the four nutrients were not statistically different between males and females (Wilcoxon rank sum test $P$-value $=0.278$ for retinol; 0.877 for vitamin A; 0.743 for cryptoxanthin; and 0.097 for copper). We observed no statistical interaction between the four nutrients and sex (Chi-squared $P$-value for interaction $=0.374$ for retinol; 0.569 for vitamin A; 0.991 for cryptoxanthin; and 0.475 for copper).

Gut microbiome and AR. Four measures of microbial alpha-diversity were not significantly different between the AR and control groups (Table 3). The microbial beta-diversity was not significantly different between the AR and control groups (PERMANOVA $P$-value $=0.141$ and 0.277 for weighted and unweighted UniFrac analysis, respectively) and between males and females (PERMANOVA $P$-value $=0.200$ and 409 for weighted and unweighted UniFrac analysis, respectively). In total, 40 genera with mean relative abundance exceeding $0.2 \%$ were included. Among them, the relative abundances of three genera (Prevotella, Bifidobacterium, and Escherichia) were significantly different between the AR and control groups $(P=0.015,0.048$, and 0.001 , respectively; Wilcoxon rank sum test).

The relative abundance of Prevotella was either zero or near zero in a large proportion of subjects (Fig. 2a): $35.5 \%$ of the AR group and $25.5 \%$ of the control group did not harbour Prevotella at all (i.e., relative abundance was $0.0 \%$ ) in their gut microbial community, whereas the relative abundance of Prevotella widely ranged in other subjects (relative abundance reached as high as 55.6\%). Compared to the first quartile (Q1) of relative abundance of Prevotella, the age- and sex-adjusted odds ratios for Q2, Q3, and Q4 were 0.67, 0.83, and 0.35, respectively (Fig. 2b). The age- and sex-adjusted odds of AR was significantly lower in Q4 than in Q1 $(P=0.004)$, but not in Q2 and Q3. A significant dose-dependent trend was observed between relative abundance of Prevotella and the age- and sex-adjusted odds of AR ( $P$ for trend $=0.017$ ).

The relative abundance of Bifidobacterium seemed to be higher in the AR group than in controls (Fig. 2c). However, dose-dependent relationship between relative abundance of Bifidobacterium and AR was not significant after the adjustment for age and sex $(P$ for trend $=0.104)$. Compared to Q1 of relative abundance of Bifidobacterium, neither of Q2, Q3, nor Q4 showed significant difference in the age- and sex-adjusted odds of AR (Fig. 2d). 
\begin{tabular}{|l|l|l|} 
Allergic rhinitis $(n=186)$ & Control $(n=106)$ & $P$-value
\end{tabular}

\begin{tabular}{|c|c|c|c|}
\hline \multicolumn{4}{|l|}{ Alpha-diversity } \\
\hline Number of OTUs observed & $295.3 \pm 98.9$ & $304.3 \pm 100.0$ & 0.412 \\
\hline Chao 1 & $518.9 \pm 206.0$ & $548.4 \pm 215.7$ & 0.224 \\
\hline ACE & $528.2 \pm 217.2$ & $557.1 \pm 221.3$ & 0.246 \\
\hline Shannon's index & $5.04 \pm 0.64$ & $5.07 \pm 0.55$ & 0.978 \\
\hline \multicolumn{4}{|c|}{ Genus-level relative abundance, \% } \\
\hline Bacteroides & $19.44 \pm 11.28$ & $18.17 \pm 11.45$ & 0.440 \\
\hline Prevotella & $6.49 \pm 13.99$ & $11.72 \pm 17.52$ & 0.015 \\
\hline Blautia & $11.20 \pm 6.14$ & $10.23 \pm 6.65$ & 0.070 \\
\hline Bifidobacterium & $10.41 \pm 10.53$ & $7.97 \pm 9.57$ & 0.048 \\
\hline Fusicatenibacter & $3.85 \pm 3.56$ & $4.15 \pm 3.95$ & 0.717 \\
\hline Faecalibacterium & $3.49 \pm 2.81$ & $3.66 \pm 3.25$ & 0.951 \\
\hline Collinsella & $4.47 \pm 5.08$ & $3.30 \pm 3.27$ & 0.072 \\
\hline Parabacteroides & $2.35 \pm 2.19$ & $3.06 \pm 3.79$ & 0.156 \\
\hline Megamonas & $2.38 \pm 5.87$ & $2.19 \pm 5.65$ & 0.859 \\
\hline Streptococcus & $2.23 \pm 4.67$ & $2.08 \pm 4.34$ & 0.224 \\
\hline Anaerostipes & $2.19 \pm 2.52$ & $1.75 \pm 2.01$ & 0.177 \\
\hline Roseburia & $1.73 \pm 2.44$ & $1.61 \pm 1.73$ & 0.585 \\
\hline Lachnoclostridium & $1.39 \pm 1.40$ & $1.58 \pm 1.73$ & 0.161 \\
\hline Holdemanella & $1.31 \pm 3.67$ & $1.08 \pm 2.35$ & 0.103 \\
\hline Dorea & $1.03 \pm 0.82$ & $0.95 \pm 0.87$ & 0.397 \\
\hline Fusobacterium & $0.80 \pm 2.69$ & $0.94 \pm 1.93$ & 0.113 \\
\hline Faecalicatena & $1.07 \pm 1.33$ & $0.89 \pm 1.19$ & 0.401 \\
\hline Eubacterium & $0.61 \pm 0.67$ & $0.84 \pm 1.22$ & 0.505 \\
\hline Catenibacterium & $0.57 \pm 2.16$ & $0.72 \pm 2.13$ & 0.310 \\
\hline Sutterella & $0.62 \pm 0.88$ & $0.65 \pm 0.74$ & 0.168 \\
\hline Phascolarctobacterium & $0.52 \pm 0.66$ & $0.65 \pm 0.89$ & 0.681 \\
\hline Megasphaera & $0.46 \pm 1.12$ & $0.63 \pm 1.51$ & 0.729 \\
\hline Prevotellamassilia & $0.20 \pm 0.96$ & $0.53 \pm 2.22$ & 0.250 \\
\hline Coprococcus & $0.54 \pm 1.09$ & $0.51 \pm 0.96$ & 0.784 \\
\hline Lactobacillus & $0.66 \pm 1.50$ & $0.48 \pm 0.84$ & 0.887 \\
\hline Faecalimonas & $0.35 \pm 0.83$ & $0.43 \pm 1.07$ & 0.376 \\
\hline Anaerobutyricum & $0.38 \pm 0.42$ & $0.42 \pm 0.45$ & 0.592 \\
\hline Mitsuokella & $0.26 \pm 1.09$ & $0.40 \pm 1.35$ & 0.578 \\
\hline Erysipelatoclostridium & $0.40 \pm 0.75$ & $0.37 \pm 0.80$ & 0.202 \\
\hline Veillonella & $0.50 \pm 1.48$ & $0.37 \pm 1.12$ & 0.815 \\
\hline Dialister & $0.30 \pm 0.72$ & $0.36 \pm 0.83$ & 0.802 \\
\hline Butyricicoccus & $0.36 \pm 0.27$ & $0.36 \pm 0.30$ & 0.838 \\
\hline Alistipes & $0.35 \pm 0.44$ & $0.32 \pm 0.39$ & 0.911 \\
\hline Clostridium & $0.43 \pm 2.72$ & $0.30 \pm 0.93$ & 0.182 \\
\hline Paraprevotella & $0.19 \pm 0.44$ & $0.28 \pm 0.59$ & 0.127 \\
\hline Romboutsia & $0.33 \pm 0.81$ & $0.28 \pm 0.45$ & 0.905 \\
\hline Escherichia & $0.62 \pm 2.60$ & $0.26 \pm 1.21$ & 0.001 \\
\hline Robinsoniella & $0.38 \pm 0.97$ & $0.26 \pm 0.56$ & 0.590 \\
\hline Lachnospira & $0.20 \pm 0.46$ & $0.24 \pm 0.49$ & 0.871 \\
\hline Parasutterella & $0.27 \pm 0.58$ & $0.18 \pm 0.41$ & 0.133 \\
\hline
\end{tabular}

Table 3. Gut microbial characteristics and allergic rhinitis. Values are shown as the mean \pm standard deviation. $P$-values were calculated using the Wilcoxon rank sum test. No adjustments were made for statistical tests shown in this table. OTU indicates operational taxonomic unit.

The AR group showed a higher relative abundance of Escherichia than controls (Fig. 2e). A clear dosedependent relationship was observed between relative abundance of Escherichia and age- and sex-adjusted odds of AR ( $P$ for trend $=0.001$; Fig. 2f). Compared to Q1, the age- and sex-adjusted odds ratios for Q2, Q3, and Q4 were $1.84,2.02$, and 2.85 , respectively. $\mathrm{Q} 4$ showed a significantly higher age- and sex-adjusted odds of AR than Q1 $(P=0.001)$, whereas Q2 and Q3 did not. 
a



e



b

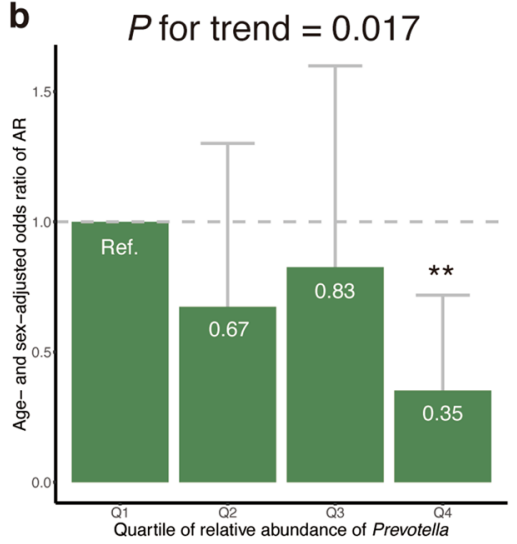

f $\quad P$ for trend $=0.001$

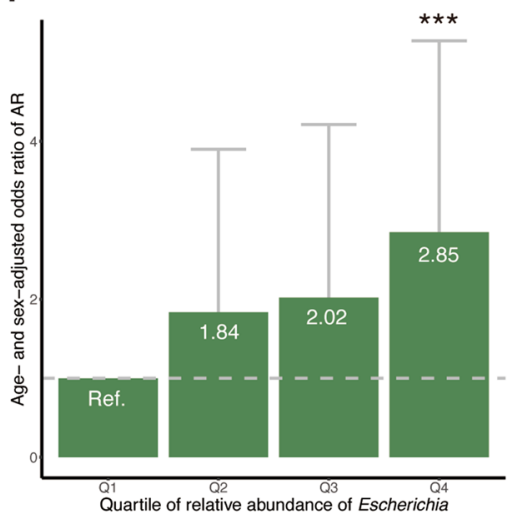

c



d $\quad P$ for trend $=0.104$

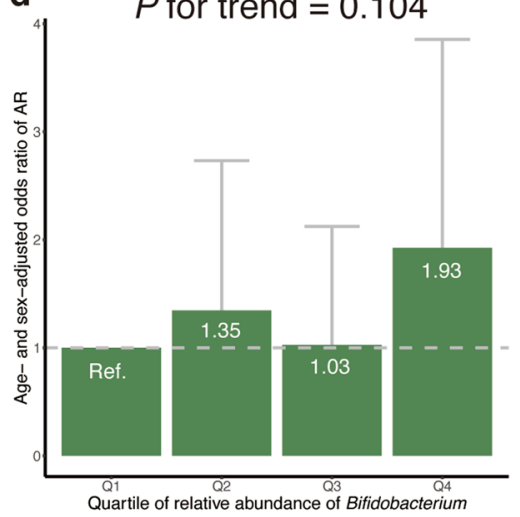

Figure 2. Gut microbial genera associated with allergic rhinitis. The distribution of relative abundance of gut microbial genera is shown for (a) Prevotella, (c) Bifidobacterium, and (e) Escherichia. Outlier data points are excluded from these plots. The age- and sex-adjusted odds ratio of allergic rhinitis is presented for (b) Prevotella, (d) Bifidobacterium, and (f) Escherichia. Error bars indicate $95 \%$ confidence interval. ' (*) indicates $P<0.05$; '**), $P<0.01$; and ${ }^{(* * *)}, P<0.001$. AR, allergic rhinitis; $\mathrm{Q}$, quartile; Ref., reference group.

No significant correlation was observed between relative abundances of Prevotella and Escherichia $(R=-0.04$; $P=0.477$; Supplementary Fig. S2). A multivariate analysis incorporating age, sex, Prevotella, and Escherichia revealed that a high relative abundance of Prevotella (Q4) and a high relative abundance of Escherichia (Q4) were independently associated with decreased and increased odds of AR, respectively $(P<0.05$; Supplementary Table S2). Bifidobacterium was not incorporated in the multivariate analysis because relative abundance of Bifdobacterium was not significantly associated with AR after adjusted for age and sex.

The ability of the microbial variables (Prevotella and Escherichia) in combination to distinguish the AR and control groups was only modest (AUC $=0.638 ; 95 \%$ confidence interval, $0.572-0.704)$. We observed no statistical difference in the relative abundance of the two genera between males and females (Wilcoxon rank sum test $P$-value $=0.307$ for Prevotella ; and 0.446 for Escherichia $)$ and no statistical interaction between the two genera and sex (Chi-squared $P$-value for interaction $=0.074$ for Prevotella; and 0.158 for Escherichia).

Combinatorial effect of dietary nutrients and gut microbiome. Significant correlation was not observed between nutritional and microbial variables except for those between retinol and Prevotella $(R=0.16$; $P=0.005)$ and between vitamin A and Prevotella $(R=0.13 ; P=0.032)$ (Supplementary Fig. S3). A multivariate analysis incorporating age, sex, four nutrients (retinol, vitamin A, cryptoxanthin, and copper), and two microbial genera (Prevotella and Escherichia) indicated that a high level of retinol intake (Q4), intermediate levels of copper intake (Q2 and Q3), a high relative abundance of Prevotella (Q4), and a high relative abundance of Escherichia (Q4) were independently associated with AR $(P<0.05$; Supplementary Table S3). A high level of cryptoxanthin $(\mathrm{Q} 4)$ was marginally associated with AR in the multivariate analysis $(P<0.10)$, whereas vitamin A was not statistically associated with the AR (Supplementary Table S3).

To investigate the combinatorial effects of dietary nutrients and gut microbiome, we re-defined nutritional groups based on the abovementioned trends observed in the association analysis. Q1-Q3 were compared to Q4 for retinol, vitamin A, and cryptoxanthin, as Q4, but not Q2 and Q3, showed a significantly lower odds of AR compared to Q1 for these three nutrients. Q1 was compared to Q2-Q4 for copper because Q2 and Q3 had a significantly lower odds of AR than Q1. Similarly, Q1-Q3 were compared to Q4 for Prevotella and Escherichia. 
a

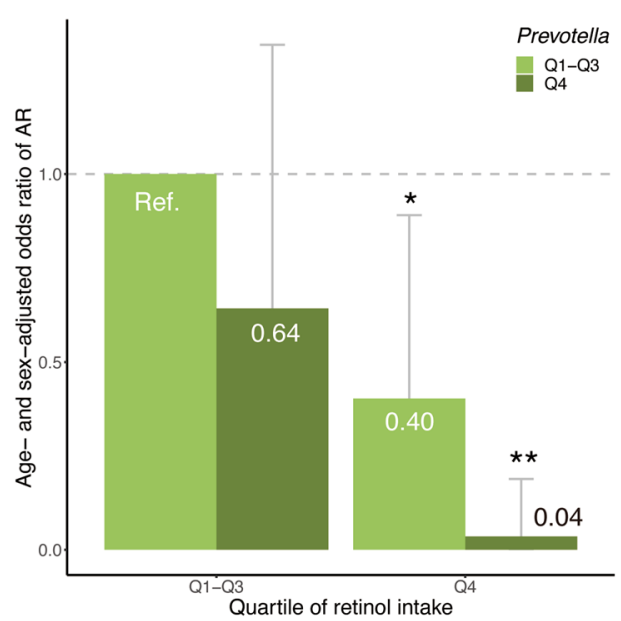

b $\quad P$ for interaction $=0.041$

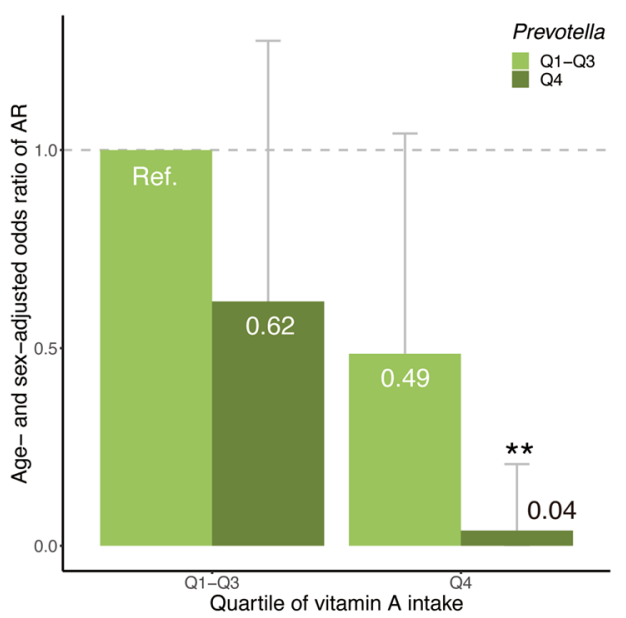

Figure 3. Significant statistical interactions between nutritional and gut microbial variables. Statistical interactions between (a) retinol and Prevotella and (b) vitamin A and Prevotella are shown. The $y$-axis indicates the age- and sex-adjusted odds ratio of allergic rhinitis. Error bars indicate $95 \%$ confidence interval. ' ${ }^{\prime}$ ' indicates $P<0.05$; and '**', $P<0.01$. AR, allergic rhinitis; $Q$, quartile; Ref., reference group.

Statistical interactions for 8 combinations of the 4 nutrients $\times 2$ microbial genera were tested. Then, significant interactions were observed between retinol and Prevotella ( $P$ for interaction $=0.048$ ) and between vitamin $A$ and Prevotella ( $P$ for interaction $=0.041)$, but not for the other 6 combinations (Supplementary Fig. S4).

The age- and sex-adjusted odds ratio of AR was 0.64 in the low or intermediate retinol intake group (Q1-Q3) with a high relative abundance of Prevotella (Q4), compared to the reference group with Q1-Q3 of retinol intake and low relative abundance of Prevotella (Q1-Q3) (Fig. 3a). The age- and sex-adjusted odds ratio of AR was 0.40 in the high retinol intake group (Q4) with low or intermediate abundance level of Prevotella (Q1-Q3) compared to the reference. In the Q4 retinol intake group with high abundance level of Prevotella (Q4), a combinatorial protective effect was observed $(P$ for interaction $=0.048$ ) the age- and sex-adjusted odds ratio for this group was 0.04 , which was 6.5 -times smaller than the expectation based on a simple multiplication that assumes no statistical interaction $(0.04<0.64 \times 0.40 \approx 0.26)$.

A similar statistical interaction was observed between vitamin A and Prevotella (Fig. 3b).

\section{Discussion}

In this study, we aimed to (i) find nutrients associated with AR; (ii) discover gut microbes associated with AR; and (iii) examine the combinatorial effect between nutrients and gut microbes on AR. Towards these ends, we recruited 186 subjects with AR symptoms and 106 controls and examined their dietary nutritional intake and composition of the gut microbiome. An association analysis suggested that retinol, vitamin A, cryptoxanthin, and copper were associated with the age- and sex-adjusted odds of AR. Furthermore, the relative abundances of Prevotella and Escherichia were associated with the age- and sex-adjusted odds of AR. Lastly, statistical interactions were observed between retinol and Prevotella as well as between vitamin A and Prevotella.

Principal dietary sources of vitamin A were retinol from animal foods and provitamin A carotenoids present in fruits, vegetables, egg yolk, and butter. As in a previous study ${ }^{16}$, we defined dietary intake of vitamin A (retinol equivalent) as the sum of retinol, $\beta$-carotene/12, $\alpha$-carotene $/ 24$, and cryptoxanthin $/ 24$. $\beta$-Carotene, $\alpha$-carotene, and cryptoxanthin are common provitamin A carotenoids, which can be converted into retinol by enzymes in the intestinal mucosa ${ }^{22}$. As expected from this definition, dietary intake levels of vitamin $A$ and retinol were highly correlated in our study population $(R=0.96)$. Vitamin $\mathrm{A}$ is essential for multiple functions in the human body, including embryonal development, good vision, epithelial differentiation, and maintenance of immune function $^{23}$, particularly in the differentiation of naïve T cells. Without retinoic acid, a metabolite of vitamin A, transforming growth factor beta (TGF- $\beta$ ) promotes the differentiation of naïve T cells into T helper 17 (Th17) cells, which are involved in inflammation, autoimmunity, and allergic disorders ${ }^{24,25}$. In the presence of retinoic acid, TGF- $\beta$ helps intestinal dendritic cells to mediate the differentiation of naïve T cells into Treg cells, which suppress autoimmune responses ${ }^{24,26}$. Natural Treg cells develop in the thymus, whereas Treg cells that develop in non-thymus tissues are called induced Treg (iTreg) cells ${ }^{27}$. iTreg cells play a crucial role in the maintenance of intestinal homeostasis, including tolerance to commensal bacteria ${ }^{28}$. In addition, vitamin A is important for gut mucosal turnover and barrier function ${ }^{29}$, intestinal IgA secretion ${ }^{30}$, and T cell homing to the intestine ${ }^{28}$. Taken together, this finding that vitamin A and retinol are associated with a decreased odds of AR may be related to the role of vitamin A in modulating intestinal immune responses.

Dietary antioxidants may protect against allergic diseases ${ }^{8}$. From this perspective, vitamin A and carotenoids are the most effective antioxidants at low oxygen tensions that are typical of human tissues ${ }^{31}$. Among the carotenoids examined in this study, only cryptoxanthin was associated with AR, whereas $\alpha$-carotene and $\beta$-carotene 
were not. As all three of these carotenoids have antioxidant activity ${ }^{32,33}$, it remains unclear whether the association of retinol, vitamin A, and cryptoxanthin with AR is related to their antioxidant activity.

Copper, an essential trace metal, is a cofactor of many redox enzymes ${ }^{34}$. As such, it is involved in iron metabolism, antioxidant activity, neuropeptide synthesis, and immune function ${ }^{35}$. Copper is essential for humans but toxic at high levels; and therefore, both copper deficiency and excess can produce adverse health effects ${ }^{36}$. Copper deficiency influences the oxidant defence system, resulting in increased oxidative damage to lipids, DNA, and proteins $^{37}$. Similarly, the toxic effects of copper at high concentrations are related to the generation of oxygen free radicals, and excess copper enhances lipid peroxidation and DNA damage ${ }^{38}$. In this study, we observed a reverse J-shaped relationship between copper and AR. This reverse J-shaped curve may be related to the anti- and pro-oxidant activities of copper at appropriate and high concentrations, respectively.

The human gut harbours hundreds of microbial species. According to Arumugam et al. ${ }^{39}$, compositions of the human gut microbiome are classified into three clusters, which are named enterotypes. The first enterotype was characterised by Bacteroides (B-type), whereas the second and third enterotypes were dominated by Prevotella (P-type) and Ruminococcus (R-type), respectively. Later studies reduced the number of enterotype clusters into two, because the B- and P-type enterotypes were consistently identified, but the identification of R-type was dependent on clustering and modelling methods ${ }^{40-42}$. Note that the concept of enterotypes has been challenged by some researchers because the B- and P-type enterotypes were not clustered separately in largescale datasets, but instead samples were distributed in a gradient across the B- and P-type groups ${ }^{43}$. Long-term dietary patterns are associated with enterotypes ${ }^{40}$. Individuals who habitually consume a Western diet high in protein and animal fat tended to have the B-type enterotype ${ }^{40}$. In contrast, vegetarian, or Mediterranean diets rich in fruits and vegetables were positively associated with P-type enterotype ${ }^{44}$. Additionally, plant-rich diets abundant in carbohydrates and fibres were associated with the P-type enterotype ${ }^{42}$. Based on these studies, it can be inferred that the relative abundance of Prevotella may reflect adherence to healthy dietary patterns. We observed a negative association between Prevotella and AR, and the association was still significant even after adjusted for AR-associated nutrients. Thus, it is unlikely that the association between Prevotella and AR is confounded by dietary patterns.

Several observational studies found that the P-type enterotype was frequently observed in rural or isolated populations, whereas the B-type enterotype was abundant in industrialised countries ${ }^{45,46}$. For example, a study of Chinese nomads showed that the prevalence of the P-type enterotype gradually decreased with the degree of urbanisation ${ }^{47}$. Given this gradient of Prevotella along with urbanisation, our finding of the association between Prevotella and AR reminds us of the microflora hypothesis of allergic diseases, which states that an unhealthy microbiota composition attributable to urbanisation or westernisation contributes to the development of allergies ${ }^{48}$. Several studies support this hypothesis. First, since the 1950s, industrialisation and urbanisation have accelerated around the world, and simultaneously, the prevalence of allergic diseases has increased in urban areas ${ }^{49}$. Second, exposures to animals, early day-care attendance, and increased number of siblings were associated with a decreased risk of allergen sensitisation ${ }^{50}$, indicating that early exposures to multiple types of microorganisms may facilitate development of the immune system ${ }^{49}$. Similarly, having older siblings was also associated with a decreased risk of AR in children ${ }^{51}$. Third, reduced exposure to microorganisms in early life is responsible for a shift in the balance between type 1 and type 2 helper $\mathrm{T}$ (Th1/Th2) cells towards the overactive Th2 arm, which stimulates IgE-mediated allergic response ${ }^{52}$. Based on the microflora hypothesis, our data indicate that the abundance of Prevotella in adults may serve as a surrogate marker for early exposure to particular microorganisms that are essential for immune system development. Alternatively, Prevotella itself may be one of the essential microorganisms for the development of immune system.

Several studies have reported the relationship between Escherichia species and allergic disorders. The intestinal relative abundance of Escherichia was higher in children with asthma or rhinitis than in controls ${ }^{53}$. The positive association between Escherichia and asthma was replicated in another study ${ }^{54}$. In infants, a high abundance of E. coli was associated with an increased risk of atopic eczema ${ }^{55}$. To our knowledge, little is known about the molecular mechanisms underlying the association between Escherichia and allergies. We expanded previous findings in children by providing evidence of a positive association between Escherichia and AR in adults.

Bisgaard et al. ${ }^{56}$ reported that bacterial diversity in the early intestinal flora 1 and 12 months after birth was inversely associated with the risk of developing allergic sensitization, peripheral blood eosinophils, and AR during the period until 6 years of age. Meanwhile, gut microbial diversity at 7 years of age was not associated with the presence of AR symptoms ${ }^{57}$. A recent study compared gut microbial diversity between $33 \mathrm{AR}$ and 31 non-AR adults (mean age was 32 years old in both groups) and showed that the alpha-diversity in AR group was significantly higher than that of the non-AR group ${ }^{58}$. Our data showed that there was no statistical difference in gut microbial diversity between AR and control groups. Further researches, including meta-analyses and systematic reviews, are required to conclude whether the alpha-diversity of adult gut microbiome is associated with the risk of AR.

We observed a statistical interaction between retinol and Prevotella as well as between vitamin A and Prevotella, suggesting that a combination of high dietary intake of retinol and carotenoids with high abundance of Prevotella may have a protective effect on the development of AR. Retinoic acid, which is derived from retinol or carotenoids, modulates the intestinal immune system ${ }^{59}$. In humans, retinoic acid is irreversibly synthesised from retinal by a host enzyme, aldehyde dehydrogenase ${ }^{60}$, which is in turn reversibly produced from retinol by alcohol dehydrogenases or retinol dehydrogenase ${ }^{60}$, or from carotenoids by $\beta$-carotene- 15 , 15 -oxygenase $1^{61}$. Interestingly, some gut microorganisms encode enzymes that are potentially involved in retinal biosynthesis ${ }^{62}$. For example, Prevotella marshii DSM 16,973 harbours a gene belonging to the $b r p / b l h$ family $^{63}$, which encodes an enzyme that produces retinal from $\beta$-carotene ${ }^{64}$. The observed statistical interaction of retinol and vitamin A with Prevotella may indicate a complex interplay of host and bacterial genes in the metabolism of retinol and carotenoids. 
The present study has several limitations. First, we searched for nutritional and gut microbial factors associated with $\mathrm{AR}$ and incorporated 42 nutrients and 40 genera in association analyses. We did not apply multiple testing correction. Therefore, our findings should be carefully interpreted. Although we found multiple candidate factors associated with AR, further studies are needed to confirm the associations. Second, our definition of AR was based on self-reported symptoms. A clinical diagnosis based on interviews, rhinoscopy, skin tests, and allergen-specific IgE tests is required for a more accurate definition. Although AR and control groups could be misclassified due to self-reporting, epidemiological studies based on self-reported AR symptoms have assumed that the misclassification was most likely nondifferential (nondifferential means unrelated to potential risk factors of interest $)^{65}$. Under the presence of nondifferential misclassification, the observed association is usually underestimated ${ }^{65,66}$. Accordingly, it is unlikely that our findings are an artefact caused by the self-reported definition of AR. Third, $~ 90 \%$ of the subjects were male. Although we observed no evidence of sex-specific effects of nutritional and microbial factors on the odds of $\mathrm{AR}$, further research is warranted to reveal whether our findings are consistent with female populations.

In conclusion, we suggest that four nutrients (retinol, vitamin A, cryptoxanthin, and copper) and two gut microbial genera (Prevotella and Escherichia) were associated with the age- and sex-adjusted odds of AR. In addition, a combinatorial protective effect of retinol and Prevotella was observed, and the age- and sex-adjusted odds of AR was 25-fold lower in subjects with a high level of dietary retinol intake and a high abundance of Prevotella compared to those with low retinol intake and a low abundance of Prevotella. Our results provide insight into the complex interplay between dietary nutrients, gut microbiome, intestinal immune systems, and the development of AR.

\section{Data availability}

The data are not available for public access because of participant privacy concerns, but are available from the corresponding author on reasonable request.

Received: 13 October 2021; Accepted: 15 February 2022

Published online: 01 March 2022

\section{References}

1. Bousquet, J. et al. Allergic rhinitis and its impact on asthma (ARIA) 2008 update (in collaboration with the World Health Organization, GA(2)LEN and AllerGen). Allergy 63(Suppl 86), 8-160. https://doi.org/10.1111/j.1398-9995.2007.01620.x (2008).

2. Wheatley, L. M. \& Togias, A. Clinical practice. Allergic rhinitis. N. Engl. J. Med. 372, 456-463. https://doi.org/10.1056/NEJMc p1412282 (2015)

3. Brozek, J. L. et al. Allergic rhinitis and its impact on asthma (ARIA) guidelines-2016 revision. J. Allergy Clin. Immunol. 140, 950-958. https://doi.org/10.1016/j.jaci.2017.03.050 (2017).

4. Bjermer, L., Westman, M., Holmstrom, M. \& Wickman, M. C. The complex pathophysiology of allergic rhinitis: Scientific rationale for the development of an alternative treatment option. Allergy Asthma Clin. Immunol. 15, 24. https://doi.org/10.1186/s13223018-0314-1 (2019).

5. McKeever, T. M. \& Britton, J. Diet and asthma. Am. J. Respir. Crit. Care Med. 170, 725-729. https://doi.org/10.1164/rccm.200405611PP (2004).

6. Garcia-Larsen, V. et al. Asthma and dietary intake: An overview of systematic reviews. Allergy 71, 433-442. https://doi.org/10. 1111/all.12800 (2016).

7. Moreno-Macias, H. \& Romieu, I. Effects of antioxidant supplements and nutrients on patients with asthma and allergies. J. Allergy Clin. Immunol. 133, 1237-1244; quiz 1245. https://doi.org/10.1016/j.jaci.2014.03.020 (2014).

8. Nurmatov, U., Devereux, G. \& Sheikh, A. Nutrients and foods for the primary prevention of asthma and allergy: Systematic review and meta-analysis. J. Allergy Clin. Immunol. 127(724-733), e721-730. https://doi.org/10.1016/j.jaci.2010.11.001 (2011).

9. Pascal, M. et al. Microbiome and allergic diseases. Front. Immunol. 9, 1584. https://doi.org/10.3389/fimmu.2018.01584 (2018).

10. Hirata, S. I. \& Kunisawa, J. Gut microbiome, metabolome, and allergic diseases. Allergol. Int. 66, 523-528. https://doi.org/10.1016/j. alit.2017.06.008 (2017).

11. McKenzie, C., Tan, J., Macia, L. \& Mackay, C. R. The nutrition-gut microbiome-physiology axis and allergic diseases. Immunol. Rev. 278, 277-295. https://doi.org/10.1111/imr.12556 (2017).

12. Honda, T. et al. Association between information and communication technology use and ocular axial length elongation among middle-aged male workers. Sci. Rep. 9, 17489. https://doi.org/10.1038/s41598-019-53423-8 (2019).

13. Matsushita, Y. et al. Associations of visceral and subcutaneous fat areas with the prevalence of metabolic risk factor clustering in 6,292 Japanese individuals: The Hitachi Health Study. Diabetes Care 33, 2117-2119. https://doi.org/10.2337/dc10-0120 (2010).

14. Matsushita, Y. et al. Effect of longitudinal changes in visceral fat area and other anthropometric indices to the changes in metabolic risk factors in Japanese men: The Hitachi Health Study. Diabetes Care 35, 1139-1143. https://doi.org/10.2337/dc11-1320 (2012).

15. Kobayashi, S. et al. Comparison of relative validity of food group intakes estimated by comprehensive and brief-type self-administered diet history questionnaires against $16 \mathrm{~d}$ dietary records in Japanese adults. Public Health Nutr. 14, 1200-1211. https://doi. org/10.1017/S1368980011000504 (2011).

16. Kobayashi, S. et al. Both comprehensive and brief self-administered diet history questionnaires satisfactorily rank nutrient intakes in Japanese adults. J. Epidemiol. 22, 151-159. https://doi.org/10.2188/jea.je20110075 (2012).

17. Watanabe, T. Food composition tables of japan and the nutrient table/database. J. Nutr. Sci. Vitaminol. (Tokyo) 61(Suppl), S25-S27. https://doi.org/10.3177/jnsv.61.S25 (2015).

18. Willett, W. \& Stampfer, M. J. Total energy intake: implications for epidemiologic analyses. Am. J. Epidemiol. 124, 17-27. https:// doi.org/10.1093/oxfordjournals.aje.a114366 (1986).

19. Kim, S. W. et al. Robustness of gut microbiota of healthy adults in response to probiotic intervention revealed by high-throughput pyrosequencing. DNA Res. 20, 241-253. https://doi.org/10.1093/dnares/dst006 (2013).

20. Lozupone, C. A. \& Knight, R. Species divergence and the measurement of microbial diversity. FEMS Microbiol. Rev. 32, 557-578. https://doi.org/10.1111/j.1574-6976.2008.00111.x (2008).

21. Robin, X. et al. pROC: An open-source package for R and S+ to analyze and compare ROC curves. BMC Bioinform. 12, 77. https:// doi.org/10.1186/1471-2105-12-77 (2011).

22. Nagao, A. Oxidative conversion of carotenoids to retinoids and other products. J. Nutr. 134, 237S-240S. https://doi.org/10.1093/ jn/134.1.237S (2004). 
23. Tanumihardjo, S. A. Vitamin A: Biomarkers of nutrition for development. Am. J. Clin. Nutr. 94, 658S-665S. https://doi.org/10. 3945/ajcn.110.005777 (2011).

24. Mucida, D. et al. Reciprocal TH17 and regulatory T cell differentiation mediated by retinoic acid. Science 317, 256-260. https:// doi.org/10.1126/science.1145697 (2007).

25. Oboki, K., Ohno, T., Saito, H. \& Nakae, S. Th17 and allergy. Allergol Int. 57, 121-134. https://doi.org/10.2332/allergolint.R-07-160 (2008).

26. Sun, C. M. et al. Small intestine lamina propria dendritic cells promote de novo generation of Foxp3 $\mathrm{T}$ reg cells via retinoic acid. J. Exp. Med. 204, 1775-1785. https://doi.org/10.1084/jem.20070602 (2007).

27. Sakaguchi, S., Miyara, M., Costantino, C. M. \& Hafler, D. A. FOXP3+ regulatory T cells in the human immune system. Nat. Rev. Immunol. 10, 490-500. https://doi.org/10.1038/nri2785 (2010).

28. Ross, A. C. Vitamin A and retinoic acid in T cell-related immunity. Am. J. Clin. Nutr. 96, 1166S-1172S. https://doi.org/10.3945/ ajcn.112.034637 (2012).

29. Ziegler, T. R., Evans, M. E., Fernandez-Estivariz, C. \& Jones, D. P. Trophic and cytoprotective nutrition for intestinal adaptation, mucosal repair, and barrier function. Annu. Rev. Nutr. 23, 229-261. https://doi.org/10.1146/annurev.nutr.23.011702.073036 (2003).

30. Gangopadhyay, N. N., Moldoveanu, Z. \& Stephensen, C. B. Vitamin A deficiency has different effects on immunoglobulin A production and transport during influenza A infection in BALB/c mice. J. Nutr. 126, 2960-2967. https://doi.org/10.1093/jn/126. 12.2960 (1996).

31. Palace, V. P., Khaper, N., Qin, Q. \& Singal, P. K. Antioxidant potentials of vitamin A and carotenoids and their relevance to heart disease. Free Radic. Biol. Med. 26, 746-761. https://doi.org/10.1016/s0891-5849(98)00266-4 (1999).

32. Paiva, S. A. \& Russell, R. M. Beta-carotene and other carotenoids as antioxidants. J. Am. Coll. Nutr. 18, 426-433. https://doi.org/ 10.1080/07315724.1999.10718880 (1999).

33. Lorenzo, Y. et al. The carotenoid beta-cryptoxanthin stimulates the repair of DNA oxidation damage in addition to acting as an antioxidant in human cells. Carcinogenesis 30, 308-314. https://doi.org/10.1093/carcin/bgn270 (2009).

34. Scheiber, I., Dringen, R. \& Mercer, J. F. Copper: Effects of deficiency and overload. Met. Ions Life Sci. 13, 359-387. https://doi.org/ 10.1007/978-94-007-7500-8_11 (2013).

35. Bost, M. et al. Dietary copper and human health: Current evidence and unresolved issues. J. Trace Elem. Med. Biol. 35, 107-115. https://doi.org/10.1016/j.jtemb.2016.02.006 (2016).

36. Stern, B. R. et al. Copper and human health: Biochemistry, genetics, and strategies for modeling dose-response relationships. J. Toxicol. Environ. Health B Crit. Rev. 10, 157-222. https://doi.org/10.1080/10937400600755911 (2007).

37. Uriu-Adams, J. Y. \& Keen, C. L. Copper, oxidative stress, and human health. Mol. Aspects Med. 26, 268-298. https://doi.org/10. 1016/j.mam.2005.07.015 (2005).

38. Bremner, I. Manifestations of copper excess. Am. J. Clin. Nutr. 67, 1069S-1073S. https://doi.org/10.1093/ajcn/67.5.1069S (1998).

39. Arumugam, M. et al. Enterotypes of the human gut microbiome. Nature 473, 174-180. https://doi.org/10.1038/nature09944 (2011).

40. Wu, G. D. et al. Linking long-term dietary patterns with gut microbial enterotypes. Science 334, 105-108. https://doi.org/10.1126/ science.1208344 (2011).

41. Costea, P. I. et al. Enterotypes in the landscape of gut microbial community composition. Nat. Microbiol. 3, 8-16. https://doi.org/ 10.1038/s41564-017-0072-8 (2018).

42. Christensen, L., Roager, H. M., Astrup, A. \& Hjorth, M. F. Microbial enterotypes in personalized nutrition and obesity management. Am. J. Clin. Nutr. 108, 645-651. https://doi.org/10.1093/ajen/nqy175 (2018).

43. Gorvitovskaia, A., Holmes, S. P. \& Huse, S. M. Interpreting Prevotella and Bacteroides as biomarkers of diet and lifestyle. Microbiome 4, 15. https://doi.org/10.1186/s40168-016-0160-7 (2016).

44. De Filippis, F. et al. High-level adherence to a Mediterranean diet beneficially impacts the gut microbiota and associated metabolome. Gut 65, 1812-1821. https://doi.org/10.1136/gutjnl-2015-309957 (2016).

45. Precup, G. \& Vodnar, D. C. Gut Prevotella as a possible biomarker of diet and its eubiotic versus dysbiotic roles: A comprehensive literature review. Br. J. Nutr. 122, 131-140. https://doi.org/10.1017/S0007114519000680 (2019).

46. De Filippo, C. et al. Diet, environments, and gut microbiota. A preliminary investigation in children living in rural and urban Burkina Faso and Italy. Front Microbiol. 8, 1979. https://doi.org/10.3389/fmicb.2017.01979 (2017).

47. Li, J. et al. A metagenomic approach to dissect the genetic composition of enterotypes in Han Chinese and two Muslim groups. Syst. Appl. Microbiol. 41, 1-12. https://doi.org/10.1016/j.syapm.2017.09.006 (2018).

48. Shreiner, A., Huffnagle, G. B. \& Noverr, M. C. The, "Microflora Hypothesis" of allergic disease. Adv. Exp. Med. Biol. 635, 113-134. https://doi.org/10.1007/978-0-387-09550-9_10 (2008).

49. Scudellari, M. News Feature: Cleaning up the hygiene hypothesis. Proc. Natl. Acad. Sci. USA 114, 1433-1436. https://doi.org/10. 1073/pnas.1700688114 (2017).

50. Stiemsma, L. T., Reynolds, L. A., Turvey, S. E. \& Finlay, B. B. The hygiene hypothesis: Current perspectives and future therapies. Immunotargets Ther. 4, 143-157. https://doi.org/10.2147/ITT.S61528 (2015).

51. Strachan, D. P. Hay fever, hygiene, and household size. BMJ 299, 1259-1260. https://doi.org/10.1136/bmj.299.6710.1259 (1989).

52. Deo, S. S., Mistry, K. J., Kakade, A. M. \& Niphadkar, P. V. Role played by Th2 type cytokines in IgE mediated allergy and asthma. Lung India 27, 66-71. https://doi.org/10.4103/0970-2113.63609 (2010).

53. Chiu, C. Y. et al. Gut microbial dysbiosis is associated with allergen-specific IgE responses in young children with airway allergies. World Allergy Organ J. 12, 100021. https://doi.org/10.1016/j.waojou.2019.100021 (2019).

54. Chiu, C. Y. et al. Cross-talk between airway and gut microbiome links to IgE responses to house dust mites in childhood airway allergies. Sci. Rep. 10, 13449. https://doi.org/10.1038/s41598-020-70528-7 (2020).

55. Penders, J. et al. Molecular fingerprinting of the intestinal microbiota of infants in whom atopic eczema was or was not developing. Clin. Exp. Allergy 36, 1602-1608. https://doi.org/10.1111/j.1365-2222.2006.02599.x (2006).

56. Bisgaard, H. et al. Reduced diversity of the intestinal microbiota during infancy is associated with increased risk of allergic disease at school age. J. Allergy Clin. Immunol. 128(646-652), e641-645. https://doi.org/10.1016/j.jaci.2011.04.060 (2011).

57. Abrahamsson, T. R. et al. Low gut microbiota diversity in early infancy precedes asthma at school age. Clin. Exp. Allergy 44, 842-850. https://doi.org/10.1111/cea.12253 (2014).

58. Zhu, L. et al. Gut microbial characteristics of adult patients with allergy rhinitis. Microb. Cell Fact 19, 171. https://doi.org/10.1186/ s12934-020-01430-0 (2020).

59. Grizotte-Lake, M. et al. Commensals suppress intestinal epithelial cell retinoic acid synthesis to regulate interleukin-22 activity and prevent microbial dysbiosis. Immunity 49, 1103-1115 e1106. https://doi.org/10.1016/j.immuni.2018.11.018 (2018).

60. Kedishvili, N. Y. Enzymology of retinoic acid biosynthesis and degradation. J. Lipid Res. 54, 1744-1760. https://doi.org/10.1194/ jlr.R037028 (2013).

61. Eroglu, A. \& Harrison, E. H. Carotenoid metabolism in mammals, including man: formation, occurrence, and function of apocarotenoids. J. Lipid Res. 54, 1719-1730. https://doi.org/10.1194/jlr.R039537 (2013).

62. Srinivasan, K. \& Buys, E. M. Insights into the role of bacteria in vitamin A biosynthesis: Future research opportunities. Crit. Rev. Food Sci. Nutr. 59, 3211-3226. https://doi.org/10.1080/10408398.2018.1546670 (2019).

63. Culligan, E. P., Sleator, R. D., Marchesi, J. R. \& Hill, C. Metagenomic identification of a novel salt tolerance gene from the human gut microbiome which encodes a membrane protein with homology to a brp/blh-family beta-carotene 15,15'-monooxygenase. PLoS ONE 9, e103318. https://doi.org/10.1371/journal.pone.0103318 (2014). 
64. Kim, Y. S., Kim, N. H., Yeom, S. J., Kim, S. W. \& Oh, D. K. In vitro characterization of a recombinant Blh protein from an uncultured marine bacterium as a beta-carotene 15,15'-dioxygenase. J. Biol. Chem. 284, 15781-15793. https://doi.org/10.1074/jbc.M109. 002618 (2009).

65. Mai, X. M., Chen, Y., Camargo, C. A. Jr. \& Langhammer, A. Serum 25-hydroxyvitamin D levels and self-reported allergic rhinitis in Norwegian adults: The HUNT Study. Allergy 69, 488-493. https://doi.org/10.1111/all.12365 (2014).

66. Gilbert, R. et al. Misclassification of outcome in case-control studies: Methods for sensitivity analysis. Stat. Methods Med. Res. 25, 2377-2393. https://doi.org/10.1177/0962280214523192 (2016).

\section{Acknowledgements}

The authors thank the Mr. Mitsunori Yasugi in the Hitachi Health Care Center, Hitachi Ltd. for his cooperation in collecting the faecal samples and clinical data from the study participants. This research did not receive any specific grant from funding agencies in either the public, commercial, or not-for-profit sectors.

\section{Author contributions}

Y.S., M.S., W.S., and T.H. wrote the manuscript. T.N. oversaw recruitment and clinical data management. Y.S., F.H., and T.H. managed the nutritional data. W.S. and Y.O. performed library preparation, sequencing, and bioinformatic analysis to obtain gut microbiome data. Y.S. and T.H. performed the statistical analysis. M.S., E.K., and M.H. supervised the work. Y.S., F.H., M.S., T.N., and M.H. designed and coordinated the project. All authors commented on and approved the manuscript.

\section{Competing interests}

Y.S. and M.S. are employees of Hitachi High-Tech Co. F.H. is an employee of Hitachi Ltd. T.H. is a board member of Genome Analytics Japan Inc. The other authors declare that they have no conflicts of interest.

\section{Additional information}

Supplementary Information The online version contains supplementary material available at https://doi.org/ 10.1038/s41598-022-07398-8.

Correspondence and requests for materials should be addressed to Y.S.

Reprints and permissions information is available at www.nature.com/reprints.

Publisher's note Springer Nature remains neutral with regard to jurisdictional claims in published maps and institutional affiliations.

(c) (i) Open Access This article is licensed under a Creative Commons Attribution 4.0 International License, which permits use, sharing, adaptation, distribution and reproduction in any medium or format, as long as you give appropriate credit to the original author(s) and the source, provide a link to the Creative Commons licence, and indicate if changes were made. The images or other third party material in this article are included in the article's Creative Commons licence, unless indicated otherwise in a credit line to the material. If material is not included in the article's Creative Commons licence and your intended use is not permitted by statutory regulation or exceeds the permitted use, you will need to obtain permission directly from the copyright holder. To view a copy of this licence, visit http://creativecommons.org/licenses/by/4.0/.

(C) The Author(s) 2022 